\title{
Development of a Superconducting Heat Switch for an Ideal Integrating Bolometer
}

\author{
Mason A. Mok ${ }^{1,2}$ \\ Edgar R. Canavan (P.I.) $)^{2}$ \\ ${ }^{1}$ University of Wisconsin Department of Mechanical Engineering, Madison, WI \\ ${ }^{2}$ NASA Goddard Space Flight Center Cryogenics and Fluid Systems Branch, Greenbelt, MD
}

\begin{abstract}
Improving the sensitivity of current state-of-the-art bolometers is essential for future cosmology endeavors. Having a bolometer that is thermally isolated from its thermal sink is ideal in order to reduce phonon noise: the factor limiting bolometer sensitivity. To thermally isolate a bolometer as much as possible a pixel-scale superconducting heat switch is being developed to control the conductance between a bolometer and its thermal sink. The heat switch is comprised of a superconducting thin aluminum film surrounded by a niobium coil. Current is passed through the coil and the resulting magnetic field drives the aluminum into its high conductance normal state. Experimental data agrees well with the results of a thermal model of the device.
\end{abstract}

\section{Introduction}

The constant technological advancement of satellite-based detectors has helped discover much of what humanity knows about distant galaxies and how our universe evolved. Cooled to temperatures well below $1 \mathrm{~K}$ to optimize performance and greatly increase sensitivity, a bolometer measures the temperature rise of an optical absorber due to incident far-infrared radiation. To cool the absorber back down, a thermal strap of constant conductance is connected to a thermal reservoir held at a constant temperature $(<1 \mathrm{~K})$. The sensitivity of the bolometer is limited by phonon noise, also known as thermal fluctuation noise, which arises from the random exchange of energy between a thermal mass and its environment. The Noise Equivalent Power (NEP) of the device, caused by phon noise, is given by $4\left(\mathrm{k}_{\mathrm{B}} \mathrm{T}^{2} \mathrm{G}\right)^{1 / 2}$, where $\mathrm{k}_{\mathrm{B}}$ is the Boltzmann constant, $\mathrm{T}$ is the temperature of the bolometer and $G$ is the thermal conductance of the thermal path. Thus, sensitivity improves by reducing $G$ and therefore having greater thermal isolation between the absorber and the lowtemperature reservoir is beneficial. However, there are limitations on minimizing G. Cosmic ray hits can cause a large temperature rise in the absorber, and thermally isolating the absorber too much prevents the detector from recovering sufficiently quickly $(\mathrm{r}=\mathrm{C} / \mathrm{G}$, where $C$ is the heat capacity of the absorber). Hence, in an attempt to improve bolometer sensitively a new technology is proposed.

The focus of this study is to characterize a pixel-scale superconducting heat switch which can control the conductance of the thermal path between the absorber of a bolometer and the thermal reservoir. In the superconducting state the aluminum heat switch behaves like a non-metal (G a $\mathrm{T}^{4}$ ), however, by applying a magnetic field around the aluminum, the metal is driven into its

This opportunity was made possible through the WSGC internship program.

I would like to thank the WSGC for their support and for making my time at NASA possible.

I would also like to thank Ed Canavan, my mentor at Goddard, for his guidance and support. 
normal state ( $\mathrm{G}$ a $\mathrm{T}$ ) and the thermal conductivity increases by up to five orders of magnitude (Rose-Innes, 1976). Such a heat switch allows for great improvements in bolometer sensitivity, even in the presence of cosmic rays. The on-chip superconducting heat switch was developed at Goddard Space Flight Center and is the first known micro-scale heat switch of its kind. The chips were mounted in a cryostat and tested at temperatures ranging from $1 \mathrm{~K}$ to $100 \mathrm{mK}$. The temperature of the detector is measured using a Superconducting Quantum Interference Device (SQUID); electrical noise generated by the thermal agitation of electrons inside a gold resistor is measured and converted to a temperature using the Nyquist Formula (Li et al., 2000). Thus, no heat is generated in the noise thermometer, unlike in a resistive thermometer. By applying heat to the detector and measuring the temperature on each side of the heat switch at different drive coil currents to measure conductance, successful operation of the heat switch has been demonstrated and high switching ratios (Gon/Goff) have been observed. Improvements to the test set-up were completed by the author along with serious advancements to the testing and data acquisition process. Further hardware developments are currently underway with a new generation of IIB are slated for testing in the Fall of 2016. This work will demonstrate an innovative technology that will advance the field of cosmology.

\section{Description of the Test Device}

The Ideal Integrating Bolometer (IIB) was originally proposed by Kogut, DiPirro and Mosely in Ideal Integrating Bolometer, 2014. The concept was then developed further by E. Canavan, T. Stevenson and P. Nagler at Goddard Space Flight Center (GSFC). In the most recent endeavor several proof-of-principle devices were fabricated by the GSFC Detector systems branch using standard microfabrication techniques. (Fig. 1) shows microscope photographs of a test pixel (there are 2 per device). The window shown is $1 \mathrm{~mm} \times 1 \mathrm{~mm}$ and is fabricated on a thin silicon nitride membrane. The isolated portion in the center is $106 \mu \mathrm{m} \times 106 \mu \mathrm{m}$ and holds two $50 \mathrm{~m} \Omega$ gold resistors and one end of a gold heat strap. One resistor is used as a heater to replicate the thermal load of an incoming infrared photon and one is a part of a Johnson noise thermometer, which is described below. The isolated region is supported by four legs: Two carry $\mathrm{Nb}$ leads to the heater and thermometer, one is for support and one holds a narrow gold strip which comprises the heat switch. Three turns of $2 \mu \mathrm{m} \times 0.7 \mu \mathrm{m}$ Niobium surround a $6 \mu \mathrm{m}$ wide $8 \mathrm{~mm}$ long aluminum strip to comprise a superconducting heat switch. Current is passed through the niobium coil in order to create a magnetic field which drives the aluminum from its superconducting "off" state to its normal "on" state. It has been calculated that a current of $30 \mathrm{~mA}$ is sufficient to provide a field which exceeds the critical field in the aluminum. Nagler et al., 2016 discusses the fabrication of these devices further as well as provides details of a numerical solution of the London equations for this geometry which is used to calculate the critical current.

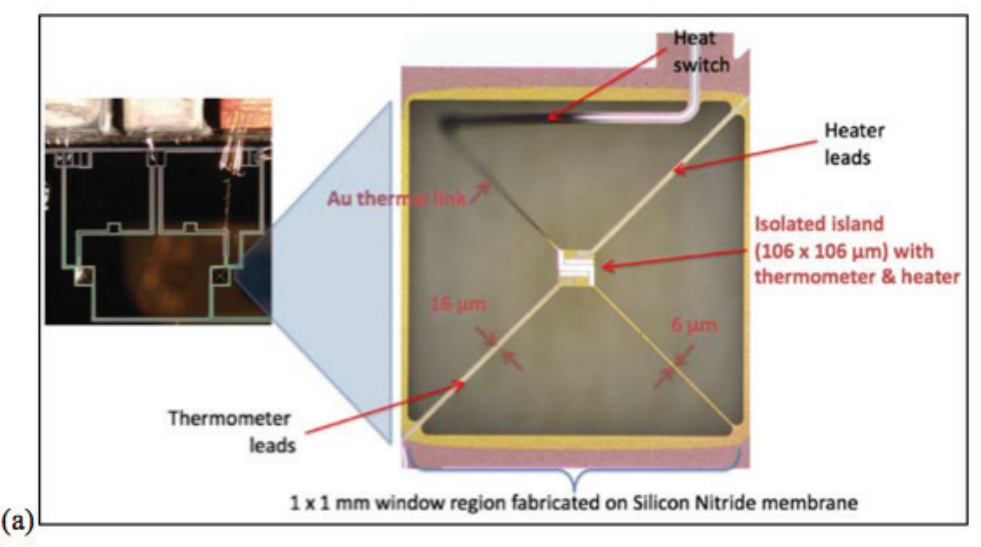


(b)
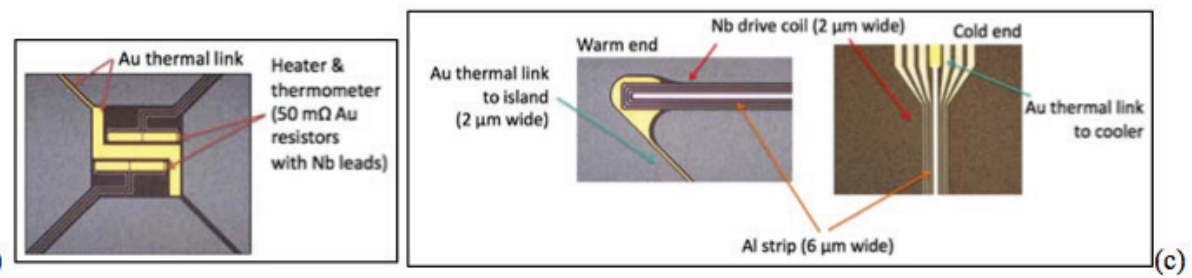

Fig 1: (a) A close-up of a test pixel. The island is supported by four legs which carry the heater and thermometer leads and the gold link for the heat switch. (b) A closer image of the isolated island showing the two resistors and the gold link. (c) Detail of the heat switch showing the overlapping gold and aluminum regions on each end of the heat switch.

\section{Experimental Set-up}

In early summer 2016 one of two remaining pixels failed. Due to the extreme sensitivity of the the pixels, a current spike in the building electrical ground may have caused this failure. Downtime during device repair allowed for improvements in the cryostat (vacuum chamber) and associated test hardware to be made. The cryostat originally consisted of a two-stage Adiabatic Demagnetization Refrigerator (ADR) (Fig.2) pre-cooled by a Cryomech PT-407 cryocooler. The approximately 1 $\mathrm{m}^{3}$ vacuum chamber was stripped down in order to allow for a new electrically isolated Cryomech PT-410 crycooler to be installed. By electrically isolating the cryostat it was hoped that each highly sensitive device would be protected against any current spike in the building ground. Furthermore, the PT-410 has greater cooling power on the first and second stage. Additionally, improvements were made to thermal joints, diode circuits were added in series with the delicate on-chip circuits to protect them from being over-charged and hardware was constructed to house additional components of the new cryocooler. For reference, the outside of the cryostat is shown in (Fig. 3). Overall, the changes to the experimental set-up reduced cool- down time and provided researches with peace-of-mind.

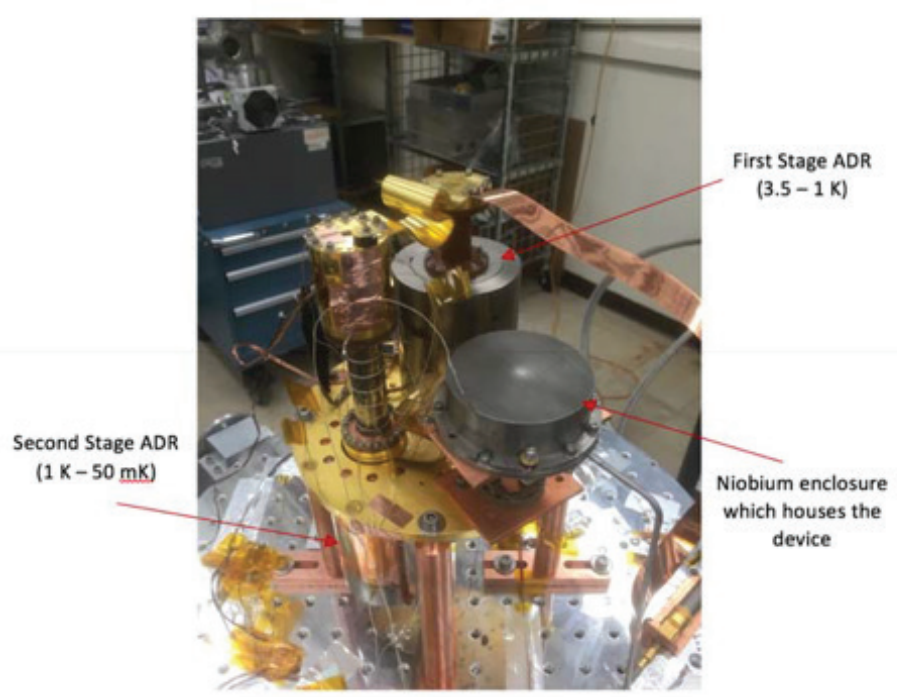

Fig 2: Adiabatic Demagnetization Refrigerator used to cool device to low temperature. 


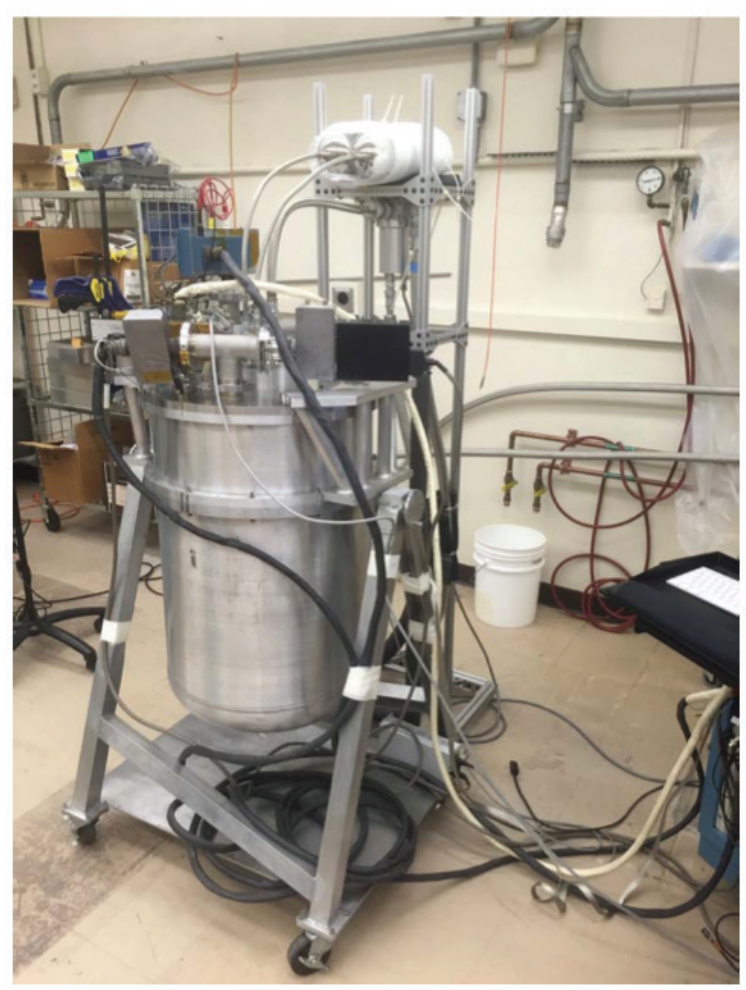

Fig 3: Outside of cryostat (vacuum chamber).

\section{Measurements}

A unique type of thermometer called a Johnson noise thermometer is used to measure the temperature of the isolated region. The thermometer uses a Superconducting Quantum Interference Device (SQUID), a low-noise amplifier, to amplify the electrical noise generated by the thermal agitation of electrons inside a $50 \mathrm{~m} \Omega$ resistor. The noise measurement is converted to a temperature using the Nyquist Formula as discussed in Li et al., 2016. One advantage of this type of thermometer is that is doesn't dump any heat at the cold end as many resistive thermometers do. However, one disadvantage is that the measurement requires 10-15 seconds to complete due to a large number of averages. Future iterations of the IIB aim to include a faster thermometer such as a magnetic penetration depth thermometer.

With the device contained in a superconducting niobium enclosure the conductance across the heat switch in the on and off states is measured at various temperatures below $1 \mathrm{~K}$ using a method similar to Rosten, et al., 2008. The conductance was measured using a technique in which a source meter applied power to the $50 \mathrm{~m} \Omega$ heater in steps and the noise thermometer read the temperature at each steady-state. Typically, 10 equal power steps were used in each conductance measurement in order to raise the detectors temperature by a total maximum of $100 \mathrm{mK}$. The slope of a linear best fit line of power vs. temperature is the conductance of the heat switch at the given temperature and applied magnetic field. A program which computes the conductance can be seen in (Fig. 4). To apply a magnetic field to the aluminum heat switch a second source meter was used to send current through the niobium coil. Conductance measurements were made at increasing magnetic fields to observe the switch from superconducting to normal behavior. The current was increased in 5 $\mathrm{mA}$ steps up to $35 \mathrm{~mA}$ to ensure the aluminum was driven entirely normal. It was experimentally 
determined that below the superconducting transition temperature of niobium the drive coil circuit had a residual resistance of $25 \mathrm{~m} \Omega$. This is most likely due to contact resistance in a screw terminal inside the niobium enclosure. Due to poor thermal conduction between the enclosure and the ADR, in early experiments the temperature in the normal state, where significant power is generated across the $25 \mathrm{~m} \Omega$ resistance, was limited. However, a high purity copper strap has been installed and the temperature is no longer limited by the inherent power generation.

Prior to Summer 2016 all data on this project was taken manually. Each change in heater power or drive coil current was made by physically changing each setting on the source meters, data was recorded by hand and ADR temperatures were changed when necessary by entering a new set point in the ADR controller. Over summer 2016 the author was tasked with automating the entire testing process using LabVIEW in order to reduce testing time and to make it possible for an engineer to remotely run tests. A series of LabVIEW virtual instruments which work cohesively was created to control heater power, drive coil current and ADR temperature based on user specifications. It is now possible for an engineer to characterize an entire pixel with minimal human intervention and data acquisition time has been reduced from days to hours. The author also created two other novel programs: one which calculates the entropy of each ADR stage and the heat load absorbed by each stage in real-time and one that can be used to "recycle" the ADR (essentially recharge with current) when the cooling power drops below a user-specified value. A sample of a program which is used to step heater power and drive coil current and plots conduction vs drive current can be seen below in (Fig. 4).

\section{Results and Discussion}

The goal of the authors internship period was to collect data using a new device (referred to as device 2 ) in a robust manner and verify results previously

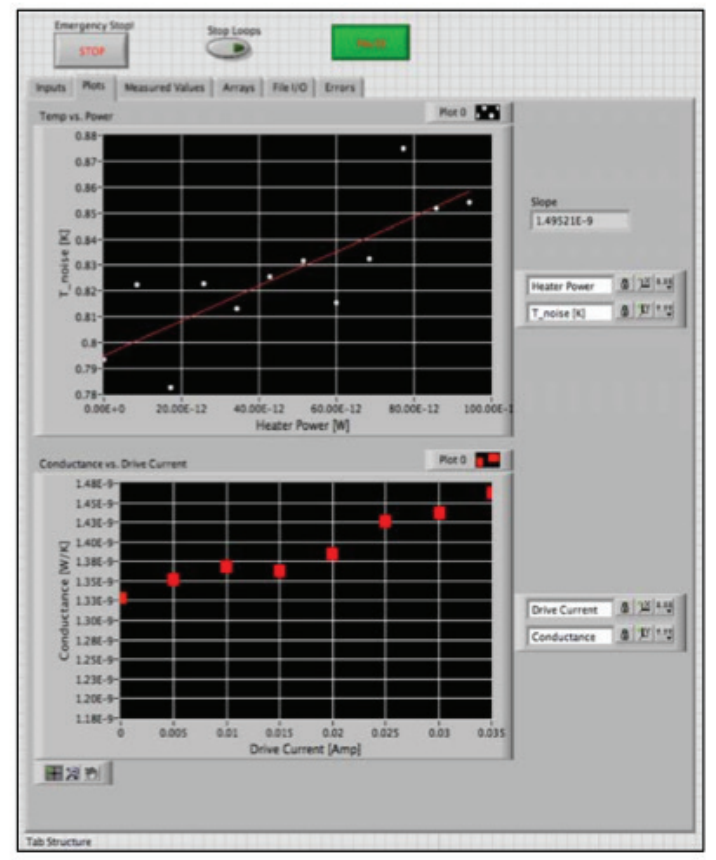

Fig 4: Sample of LabVIEW programs which automates heat switch characterization. The conductance of the heat switch is computed as the nverse slope of the temperature vs. power plot. This is then plotted against applied drive current. The data shown is for a mean temperature of $830 \mathrm{mK}$. reported by Canavan (2016). The dashed and solid
lines in (Fig. 5) depict the results of a model created by Canavan which predicts the conductance of the heat switch in the normal and superconducting states. The dashed lines represent pure aluminum and the solid lines represent the actual conductance of the device considering all constituent materials such as the silicon nitride substrate which supports the device. The diamond and circle markers are data taken prior to summer 2016 and the triangle and square markers are data taken by the author. The measurements in the superconducting state match the model very well in both instances down to a temperature of $\sim 280 \mathrm{mK}$. Due to limitations of the Johnson noise thermometer, accurate measurements below this temperature were not possible. The conductance in the normal state matches the model well but starts to deviate as temperature is reduced. From 
previous measurements of electrical resistance, it has been demonstrated that an applied current of $35 \mathrm{~mA}$ is sufficient to drive the material normal at low temperature so the material should be completely normal. According to Canavan et al., 2016, "In the normal state, the gold thermal strap and the normal aluminum, which have linear temperature dependence, dominate conductance. The coupling of heat from electrons in the gold to phonons in the silicon nitride, the diffusion of phonons across the silicon nitride gap between the gold and aluminum, and the coupling between the phonons and electrons in the normal state aluminum add series resistances in the thermal path that have a temperature dependence stronger the linear." Thus, it seems that the predictions of the model are insufficient at determining conduction in the normal state.

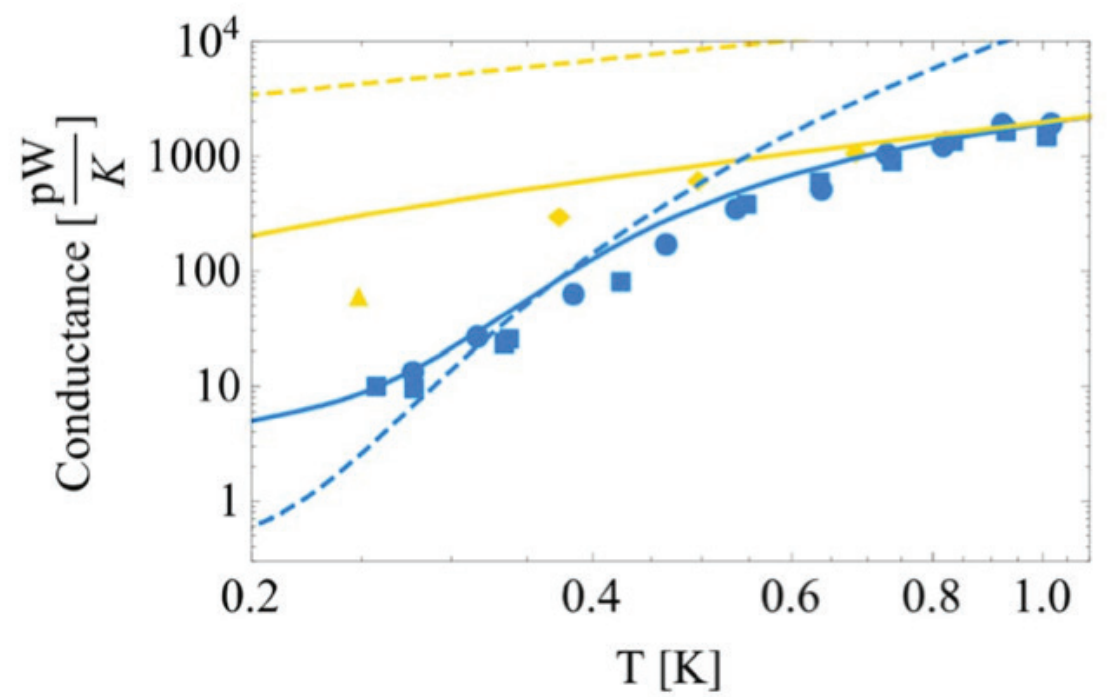

Fig. 5: Thermal Conduction of the device as a function of temperature. Circle and square shapes are taken at 0 applied field (pure superconducting) for devices 1 and 2, respectively. Diamond and triangle shapes taken at $\geq 30 \mathrm{~mA}$ for devices 1 and 2, respectively. The solid lines represent the results of a model created by E. Canavan assuming simple diffusive transport (Canavan et al., 2016). The upper and lower dashed lines represent the conduction of pure aluminum in the normal and superconducting states, respectively.

Even though the model was insufficient there is still a considerable experimental ON/OFF conduction ratio of 6 . However, compromises made during the design of this generation of devices have limited the ON/OFF conduction ratio significantly compared to the ratio or (of?) pure aluminum, which is many orders of magnitude higher. To expedite the fabrication process for a proof-of-principle test, a low cost and low risk device was created. Most notably, the drive coil was fabricated on the same wafer as the aluminum, forcing the beam that carries the heat switch to be 6 times wider than if the two components were on separate wafers. This significantly increases the conduction in the off state. Furthermore, because there is not direct contact between the gold and aluminum on the warm end of the heat switch the conductance in the on state is greatly reduced. Future generations of devices aim to achieve higher switching ratios by improving upon the design of the initial device now that the principle has been confirmed.

\section{Conclusion}

Significant progress towards a functional ideal integrating bolometer was made over summer 2016. By automating the entire testing process of a device, the time required to gather data has 
been reduced significantly. The installation of a new electrically isolated cryocooler, diode circuits and the improvement of thermal conduction across thermal joints has provided the IIB engineering team with an improved test set up. Furthermore, the programs which provide engineers with realtime data analysis and displays will benefit all engineers in the 552 branch of NASA Goddard Space Flight Center. Improvements to future generations of the IIB will include enhancements in thermometry and the application of more advanced microfabrication techniques to ensure increases in performance.

References

Canavan, E., T. R. Stevenson, P. Nagler, M. Mok. "Demonstration of a Pixel-Scale Superconducting Heat Switch for an Ideal Integrating Bolometer." Applied Superconductivity Conference 2016. Submitted for publication.

Kogut A., M. DiPirro, and S.H. Moseley, "Ideal Integrating Bolometer" New Concepts for Far-Infrared and Submillimeter Space Astronomy, Proc. of the Second Workshop on New Concepts for Far- Infrared and Submillimeter Space Astronomy, College Park, MD, USA, March 7-8, 2002, Eds. Dominic J. Benford and David T. Leisawitz, 2014.

Li, Junyun, V.a Maidanov, H. Dyball, C.p Lusher, B.p Cowan, and J. Saunders. "Current Sensing Noise Thermometry for Millikelvin Temperatures Using a DC SQUID Preamplifier." Measurement and Science Technology. 12.1 (2000): 1-15.

Nagler, P. C., E. Canavan, R. De Alba, and T. R. Stevenson. "Development of Superconducting Magnetic Heat Switches for an Ideal Integrating Bolometer.” Journal of Low Temperature Physics 184.1/2 (2016): 280-85.

Rose-Innes, A. C. Introduction to Superconductivity. 2nd ed. Oxford: Pergamon, 1976. Print.

Rostem, Karwan, Dorota M. Glowacka, David J. Goldie, and Stafford Withington. "Thermal Conductance Measurements for the Development of Ultra Low-noise Transition-edge Sensors with a New Method for Measuring the Noise Equivalent Power." Proceedings of SPIE: Millimeter and Submillimeter Detectors and Instrumentation for Astronomy 7020.70200L (2008) 